\title{
Fully bio-originated latent heat storing calcium alginate microcapsules with high coconut oil loading
}

Bence Németh ${ }^{\mathrm{a}, \mathrm{b}}$, Ágnes S. Németh ${ }^{\mathrm{a}}$, Aurél Ujhidy ${ }^{\mathrm{a}}$, Judit Tóth ${ }^{\mathrm{a}, \mathrm{b}}$, László Trif ${ }^{\mathrm{b}}$, János Gyenis ${ }^{\mathrm{a}}$, Tivadar Feczkó ${ }^{\mathrm{a}, \mathrm{b}^{*}}$

${ }^{a}$ Research Institute of Biomolecular and Chemical Engineering, University of Pannonia, Egyetem u. 10, H-8200, Veszprém, Hungary

${ }^{\mathrm{b}}$ Institute of Materials and Environmental Chemistry, Research Centre for Natural Sciences, Hungarian Academy of Sciences, Magyar tudósok körútja 2., H-1117, Budapest, Hungary

E-mails: nemeth.bence@mukki.richem.hu, ujhidy@mukki.richem.hu, szemes@mukki.richem.hu, toth@mukki.richem.hu,trif.laszlo@ttk.mta.hu,gyenis@mukki.richem.hu

* Corresponding author, e-mail: tivadar.feczko@gmail.com, phone: +36-88-624000/3508, fax: $+36-88-624038$ 


\begin{abstract}
Latent heat storing calcium alginate microcapsules were manufactured by a repeated interfacial coacervation/crosslinking method. By using a high-viscosity sodium alginate for the capsule formation, the paraffin phase change material (PCM) content was substantially enhanced related to the recently developed procedure. The maximization of PCM loading was achieved using experimental design for paraffin containing microcapsules. The microcomposites were optimized for $81.5 \%$ PCM content with uniform size of $2.20 \pm 0.14 \mathrm{~mm}$. In some applications e.g. packaging, the biodegradable character of the materials is especially beneficial, hence for the manufacture of entirely eco-friendly heat storing microcomposites coconut oil PCM was microencapsulated by the same procedure. The process originally developed and optimized for paraffin was scaled-up by two orders of magnitude, accordingly, the outstanding PCM content was reproduced also with coconut oil (average value $81.1 \%$ ). The high PCM content was reflected also in the heat storing capacity measured by differential scanning calorimetry. The easily upscalable, spherical and core/shell structured, entirely biocompatible microcapsules with thermally stable calcium alginate coating could be developed for industrial application.
\end{abstract}

Keywords: phase change materials; biocompatible microcapsules; calcium alginate; coconut oil

\title{
1. Introduction
}

Nowadays, energy storage systems are essential for reducing the fossil fuel consumption, and hence contributing to environmentally friendly energy use. Latent heat thermal energy storage is one of the most efficient ways of storing thermal energy. The latent heat energy storage in phase change materials provides much higher energy storage density than that achieved by sensible heat (Zhang et al. 2012). To improve energy utilization efficiency, the PCMs have been extensively applied in textiles, buildings, therapies, packing and spacecraft (Hu et al., 2013). Most important requirements towards PCMs are the cost-effectiveness, no corrosion to the container and easy preparation with desirable dimensions. Microencapsulated PCM can considerably enlarge the heat transfer area, reduce the PCMs reactivity with the outer environment, avoid subcooling or incongruent melting, and control the changes in the volume during melting and freezing (Zhang et al. 2012). The so-called slurry phase materials that are developed to improve the heat capacity of solar thermal collectors include phase change materials encapsulated by suspended particles, 
which remain in the liquid state of the fluid at a macroscopic scale during the phase change process (Serale et al. 2016). Form-stable PCMs can also provide some of the above mentioned benefits of PCM microparticles, however, in some applications to evade PCM leaking they should be also supplied by a protecting layer on the surface (Feczko et al. 2016). Shell material plays an important role in the heat transfer feature and mechanical strength of all the PCM entrapping microcapsules. The shell material should have sufficient structural and thermal strength to withstand numerous phase change cycles of PCM, and it must not react with the PCM (Salunkhe and Shembekar, 2012). It can also improve the compatibility of PCMs that cannot be directly immersed in certain applications, such as food storage and building cooling/heating. Although polymeric shell prevents the leakage of liquid PCMs, due to its low thermal conductivity it reduces substantially the rate of heat storage (Sarı and Karaipekli, 2007). Thus, the most important task is to decrease the mass of encapsulating material parallel with avoiding the loss of shell mechanical strength.

To our knowledge, PCM microcapsules that originate completely from biological sources have not been synthesized so far. There are some studies dealing with microencapsulation of naturally formed PCMs. Konuklu et al. (2014a,b) microencapsulated caprylic acid (octanoic acid) and decanoic acid with urea-formaldehyde-, melamine-formaldehyde- and urea-melamineformaldehyde resins by the coacervation method, and caprylic acid using polystyrene shell material by the emulsion polymerization method (Konuklu and Paksoy 2017). Palmitic acid was entrapped by some groups using organic (Doguscu et al., 2017) or inorganic (Cao et al., 2014) shells. These fatty acids are environmentally friendly, since they can be obtained from vegetable and animal oils. Decanoic acid can be derived from coconut oil, which is abundant in tropical countries. Coconut oil has suitable melting temperature, high latent heat capacity, little or no super cooling during the phase transition, non-toxicity and non-corrosivity against metal containers. In the other approach, the carrier material is of biological origin, for example chitosan, a low-price and eco-friendly polymer, was used to synthesize graphene-based carbon aerogel that was filled with 1-hexadecanol in order to prepare a form-stable PCM for thermal energy storage (Fang et al., 2017). Silk fibroin was utilized for embedment of paraffin PCM (Luo et al., 2016). n-octadecane microcapsules with gelatin-gum arabic shell were prepared by complex coacervation and crosslinking with glutaraldehyde (Li et al., 2012). As a carrier calcium alginate is favourable, e.g. it was used to entrap paraffin wax Rubitherm RT27 by a simple co- 
extrusion minifluidic device (Liang et al., 2014). n-octadecane shape stabilized with calcium alginate matrix was embedded in acrylic-based copolymer capsules (Li et al., 2013). Calcium alginate was also used to incorporate shape-stabilized phase change materials (Wang et al., 2011). Thus, in former studies exclusively either the PCM or the shell material originated from biological sources.

Bio-originated PCMs such as soybean oils, coconut oils, palm oils, and beef tallow (Jeong et al., 2013) have high latent heat of fusion, good thermal stability, and no toxicity, similarly to paraffin, and are also suitable for microencapsulation. However, most paraffins are flammable, while bio-originated PCMs have considerably higher ignition resistance. Since bio-based PCMs are fully hydrogenated, they are not sensitive to oxidation. Their melting point can be adjusted in a wide temperature range, from $-23{ }^{\circ} \mathrm{C}$ up to $78{ }^{\circ} \mathrm{C}$, hence they can be suited to various application fields in various climatic condition. They possess low vapor pressure, self-nucleating behavior, safety, and commercial availability at low cost (Kang et al., 2015). The eco-friendly shell material promotes the environmental sustainability as well, because it can be degraded into non-toxic materials, hence it becomes a competitive alternative to conventional petroleum-based polymers in various applications.

Recently, we have developed a method for preparing alginate microcapsules containing paraffin by the method of repeated interfacial coacervation/crosslinking (Németh et al., 2015). In this work, the PCM loading was enormously improved by using a high-viscosity sodium alginate for forming the capsules. Moreover, the paraffin was substituted by coconut oil in order to prepare fully eco-friendly PCM containing microcomposites. Beside the characterization of size, structure and chemical composition, thermal analysis of the microcapsules was thoroughly performed too.

\section{Material and methods}

\subsection{Materials}

Sodium alginate (viscosity at $25^{\circ} \mathrm{C}$, concentration $2 \mathrm{w} / \mathrm{v} \%$ : $950 \mathrm{mPas}$ ) was bought from Cargill (US). $\mathrm{CaCl}_{2} 2 \mathrm{H}_{2} \mathrm{O}$ was purchased from Sigma-Aldrich, paraffin with melting temperature interval $55-57{ }^{\circ} \mathrm{C}$ was kindly provided by the MOL Plc, Hungary. Coconut oil was obtained from Soya Group Ltd., Hungary. Petroleum ether (boiling temperature $60-62^{\circ} \mathrm{C}$ ) was purchased from Lach- 
Ner s.r.o., Nercetovice, Czech Republic. All chemicals were of analytical grade and were used as purchased. For preparation of all aqueous solutions distilled water was used.

\subsection{Methods}

\subsubsection{Capsule preparation}

The microcomposite preparation was optimized using paraffin PCM, and the detailed process was described in our recent study (Németh et al., 2015). Compared to this cited work it was found that the application of a sodium alginate with higher viscosity (950 mPas related to the former 14 mPas, at $25^{\circ} \mathrm{C}$, concentration $2 \%$ ) results in substantially higher PCM loading in the capsules. Due to the fact, that the process parameters also changed significantly, hence they need to be presented here (Table 1). Briefly, an oil-in-water emulsion was formed by mixing $8.1 \mathrm{~g}$ solid paraffin with $45 \mathrm{~g}$ sodium alginate solution (concentration: $2.0 \%$ ) in distilled water at $65{ }^{\circ} \mathrm{C}$. After paraffin melting, it was emulsified into the aqueous phase by sonication for $3 \times 30 \mathrm{~s}$ with Vibra cell VCX130 (Sonics and Materials Inc., Newtown, USA) sonicator using $40 \%$ of its 130 Watts maximal power. The formulated oil-in-water emulsion was dropped into a gently stirred $500 \mathrm{~g}$ solution containing $4 \% \mathrm{CaCl}_{2}$. After the gelation time $(30 \mathrm{~min})$, the particles were transferred into another $\mathrm{CaCl}_{2}$ solution of various concentrations (minimum value: $1.0 \%$, medium value: $4.0 \%$, maximum value: $7.0 \%$, ) to provide excess $\mathrm{Ca}^{2+}$ ions near the surface region of core particles. The core particles were filtered from the gelling solution and added into the gently stirred sodium alginate solution (minimum value: $0.5 \%$, medium value: $2.0 \%$, maximum value: $3.5 \%$, ) for the reaction time (minimum value: $1 \mathrm{~min}$, medium value: $7 \mathrm{~min}$, maximum value: $13 \mathrm{~min}$ ). Finally, the raw capsules were removed and rinsed three times with distilled water. To solidify the microparticles, the prepared capsules were heated, while they were rolled on a hot stainless steel plate at $125^{\circ} \mathrm{C}$ for $15 \mathrm{~min}$.

3-level 3 factors Box-Behnken experimental design was carried out by the STATISTICA ${ }^{(\mathrm{R})}$ software (Statsoft Inc. USA). 3 process variables, that is, sodium alginate- and $\mathrm{CaCl}_{2}$ concentrations and contact time were found to affect considerably the paraffin content of the PCM containing microcapsules. The experimental program (Table 1) consisted of 15 runs, including 3 repetitions in the center (indicated with capital $\mathrm{C}$ in the Table). The concentrations of the first sodium alginate solution and the first calcium chloride concentrations were kept constant. 
For the coconut oil microencapsulation the process was scaled-up. To $2.75 \mathrm{~kg} 2 \%$ sodium alginate solution at $50{ }^{\circ} \mathrm{C} 1.0 \mathrm{~kg}$ melted coconut oil was added, then it was homogenized by an industrial homogenizer (IKA Ultraturrax-40) for $10 \mathrm{~min}$ to produce a stable emulsion. The emulsion was dropped by a Büchi Encapsulator B-390 into $100 \mathrm{~L} 4 \% \mathrm{CaCl}_{2}$ solution in an airlift mixer during stirring, and left for gelation for $30 \mathrm{~min}$. The formed gel particles which serve as cores in the next manufacturing step were filtered and they were dried in a fluidized bed drier equipped with conical flow diverter insert. $1.7 \mathrm{~L}$ core was added to $17 \mathrm{~L} 1.3 \%$ sodium alginate solution, while mixing with a propeller stirrer for $10 \mathrm{~min}$, then, the reaction mixture was diluted by the same volume of distilled water. The capsules were filtered and washed by distilled water, and they were mixed in $4 \% \mathrm{CaCl}_{2}$ solution for $30 \mathrm{~min}$, then filtered and washed by distilled water. The capsules were dried at $110{ }^{\circ} \mathrm{C}$ in a fluid bed drier for $15 \mathrm{~min}$.

Table 1. Paraffin content and capsule size as a function of experimental conditions (sodium alginate- and calcium chloride concentrations, contact time) using 3 factors Box-Behnken design with 1 block, 15 runs and 3 repetitions in centrum point (C).

$\begin{array}{cccccc}\text { Run \# } & \begin{array}{c}\text { Sodium alginate } \\ \text { concentration } \\ (\%)\end{array} & \begin{array}{c}\text { Calcium chloride } \\ \text { concentration }(\%)\end{array} & \begin{array}{c}\text { Contact } \\ \text { time (min) }\end{array} & \begin{array}{c}\text { Paraffin } \\ \text { weight ratio } \\ (\%)\end{array} & \begin{array}{c}\text { Mean } \\ \text { capsule size } \\ (\mathrm{mm})\end{array} \\ & & & & & \\ 1 & 3.5 & 4.0 & 13.0 & 52.44 & 2.29 \pm 0.13 \\ 2 & 3.5 & 4.0 & 1.0 & 71.76 & 2.19 \pm 0.13 \\ 3 & 2.0 & 1.0 & 1.0 & 78.22 & 2.24 \pm 0.19 \\ 4 & 3.5 & 1.0 & 7.0 & 72.03 & 2.29 \pm 0.21 \\ 5 & 0.5 & 4.0 & 1.0 & 81.53 & 2.17 \pm 0.17 \\ 6 & 0.5 & 1.0 & 7.0 & 75.97 & 2.28 \pm 0.17 \\ 7 \mathrm{C} & 2.0 & 4.0 & 7.0 & 62.28 & 2.27 \pm 0.12 \\ 8 & 2.0 & 1.0 & 13.0 & 69.18 & 2.22 \pm 0.17 \\ 9 & 2.0 & 7.0 & 1.0 & 69.10 & 2.27 \pm 0.12 \\ 10 \mathrm{C} & 2.0 & 4.0 & 7.0 & 59.21 & 2.15 \pm 0.10 \\ 11 \mathrm{C} & 2.0 & 4.0 & 7.0 & 58.84 & 2.12 \pm 0.14 \\ 12 & 0.5 & 4.0 & 13.0 & 64.10 & 2.01 \pm 0.10 \\ 13 & 2.0 & 7.0 & 13.0 & 52.71 & 2.21 \pm 0.13 \\ 14 & 0.5 & 7.0 & 7.0 & 67.16 & 2.19 \pm 0.16 \\ 15 & 3.5 & 7.0 & 7.0 & 40.69 & 2.12 \pm 0.13\end{array}$

\subsubsection{Paraffin content, size and morphology}

To analyze the paraffin content $0.1 \mathrm{~g}$ microcapsules were ground with micro-ball mill (Narva Vibrator), then, the PCM was extracted by $20 \mathrm{ml}$ petroleum ether and vacuum filtered. The 
petroleum ether was evaporated in vacuum (Heidolph Laborota 4001) and the paraffin was weighed to calculate its percentage in the capsules.

The diameter of 50 particles per sample was measured by optical microscope (Carl Zeiss). The average values and standard deviations were calculated. The morphology and cross-section of the particles were investigated after vacuum-coating with palladium and examined by a Zeiss EVO 40 scanning electron microscope.

\subsubsection{Thermogravimetric and differential scanning calorimetry measurements}

Thermogravimetric measurements were carried out on a Setaram LabsysEvo thermal analyzer, in flowing high purity synthetic air atmosphere (flow rate $90 \mathrm{~mL} / \mathrm{min}$ ), with a constant heating rate of $15^{\circ} \mathrm{C} / \mathrm{min}$. The samples were weighed into standard $100 \mu \mathrm{L}$ platinum crucibles, and were analyzed in the $30-1000{ }^{\circ} \mathrm{C}$ temperature interval. The results were processed by using Calisto Processing software (ver. 1.49), whereas from every measurement a previously recorded baseline was subtracted.

The heat storing properties of the microcapsules were determined by a Setaram $\mu$ DSC3evo differential scanning calorimeter (DSC). Each sample was weighed directly into the calorimeter's batch vessel and was cycled two times in the temperature range of $-10-+45^{\circ} \mathrm{C}$, by a scanning speed of $0.2^{\circ} \mathrm{C} / \mathrm{min}$. The results were processed by using Calisto Processing software (ver. 1.49), corresponding melting/freezing enthalpies, and the onset temperatures were determined using the baseline integration method (Tangential sigmoid baseline type).

\section{Results and discussion}

\subsection{Paraffin content optimization}

Because, due to the applied procedure, the size of the obtained microcapsules were not varied significantly with the variation of the studied process parameters the variation of paraffin content was analysed as a function of most important process variables which were proved to play the most important role in our recent study (Németh et al., 2015), i.e. sodium alginate concentration $(\%), \mathrm{CaCl}_{2}$ concentration (\%) and contact time (min).

The Pareto chart (Fig. 1) shows that the paraffin content was most substantially determined by the $\mathrm{CaCl}_{2}$ concentration and the contact time (linear influences L). Sodium alginate concentration 
was also a key parameter (linear influence). The cross-effects of the concentrations also strengthened their even high influence. The contact time effect was also enhanced by its quadratic (Q) term. Increasing the value of each parameter would decrease the paraffin content of the microcapsules by increasing the weight of the capsule shell. Each of the variables exerted statistically significant impact on the paraffin concentration of the microcapsules with a significance level well below the $\mathrm{p}=0.05$ value.

(2) Calcium chloride concentration (L)

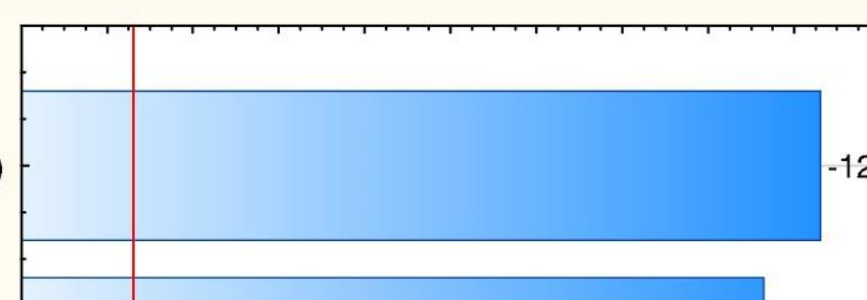

(3) Contact time, $\min (L)$

(1) Sodium alginate concentration \%(L)

(3) Contact time, $\min (\mathrm{Q})$

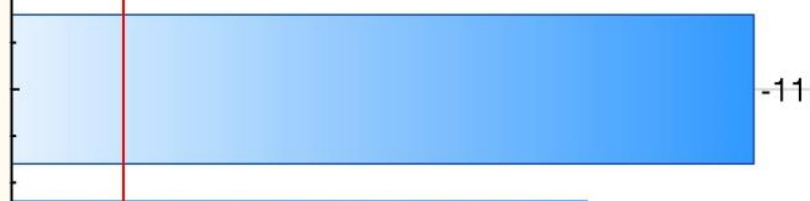
$-11.6418$

1Lby2L

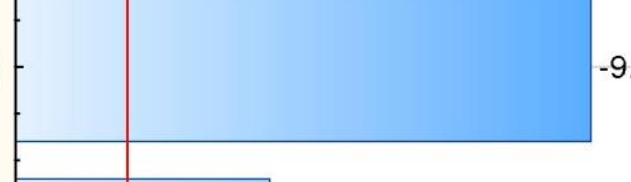

$-9.70589$ $-5.9655$

$\mathrm{p}=0,05$

$-5.1862$

Standardized Effect Estimate (Absolute Value)

Fig. 1. Influence of process variables on paraffin content discarding the non-significant effects.

3-D diagrams are suitable to draw the effects of two variables on the paraffin loading. Decreasing either the $\mathrm{CaCl}_{2}$ - or sodium alginate concentration to the medium value the paraffin content increases enormously (Fig.2), that is, the weight of capsule shell decreases, nevertheless, this tendency is decelerated considerably, when any of these concentrations reaches excessively low value, which prevents formation of suitable amount of wall material. If any of the $\mathrm{CaCl}_{2^{-}}$or sodium alginate concentration is maximal, the paraffin content increases with the other growing concentration due to the increasing coating mass. 


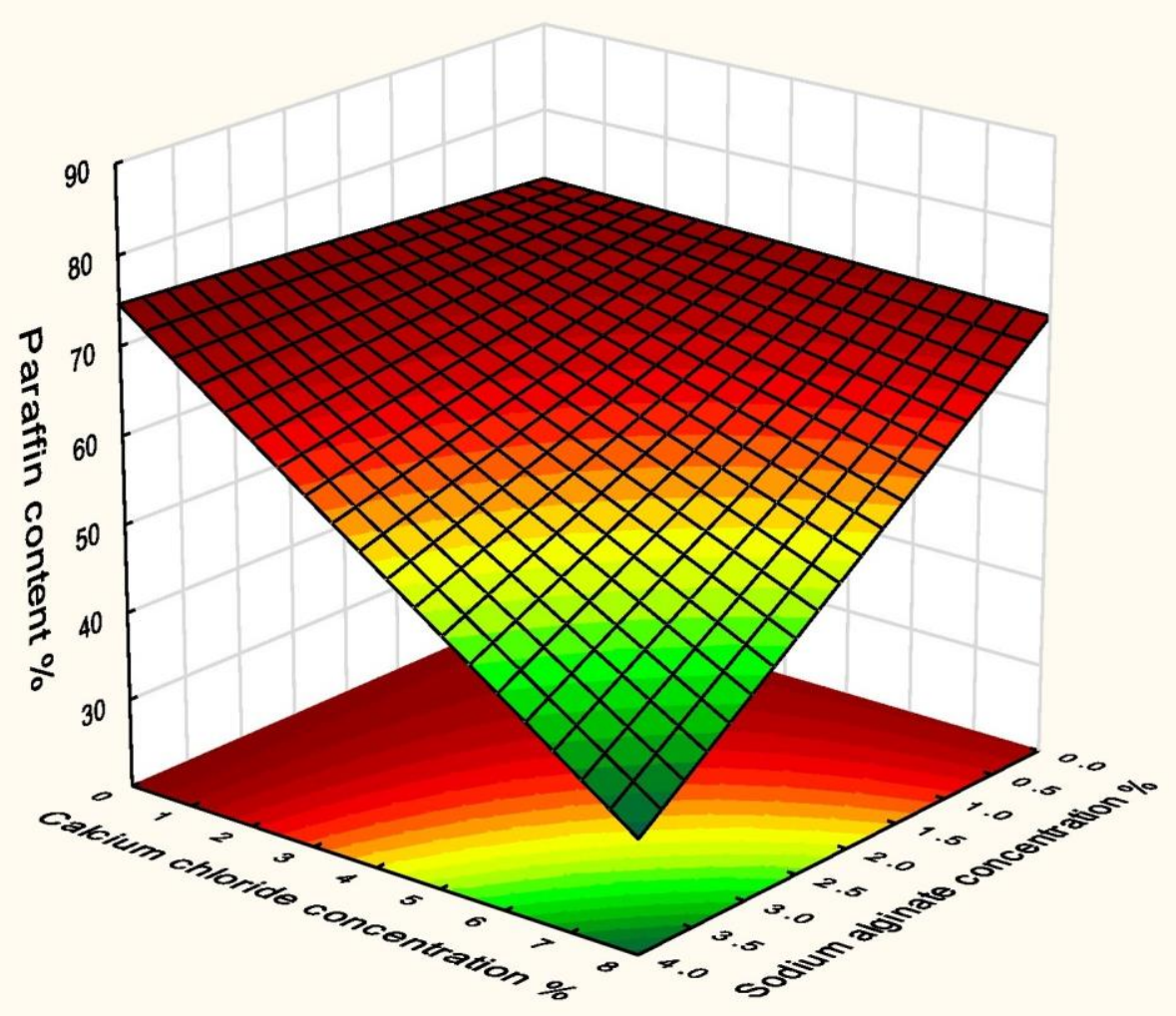

Fig. 2. Variation of paraffin content as a function of sodium alginate- and $\mathrm{CaCl}_{2}$ concentrations at the medium value $(7.0 \mathrm{~min})$ of contact time.

Throughout one of the adjusted concentrations is minimal (insufficient), the other component concentration influences slightly the final paraffin content, since the coating process can go on to a certain limit resulting in mechanically instable capsule formation. At constant $\mathrm{CaCl}_{2^{-}}$or (Fig. 3) sodium alginate (Fig.4) concentrations (medium values) increasing the contact time and the $\mathrm{CaCl}_{2^{-}}$or the sodium alginate concentration the paraffin content of the product decreases quickly, then its reduction slows down, at the same time the weight of capsule coating increases following the same tendency. The dependence on the contact time is non-linear, but it is modified according to a quadratic term. The effect of contact time is demonstrated by fixing either of the component concentrations (Fig. 3 and Fig. 4). 


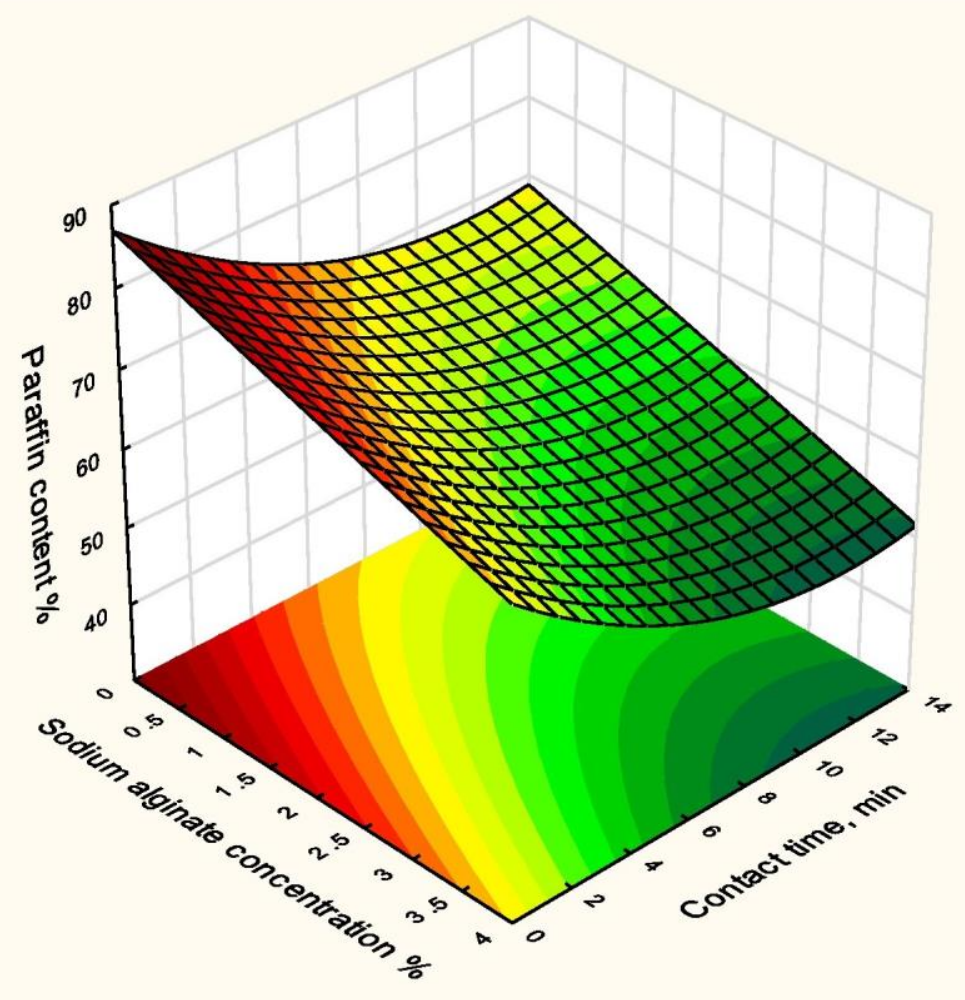

Fig. 3. Variation of paraffin content as a function of sodium alginate concentration and contact time at the medium value (4.0\%) of $\mathrm{CaCl}_{2}$ concentration. 


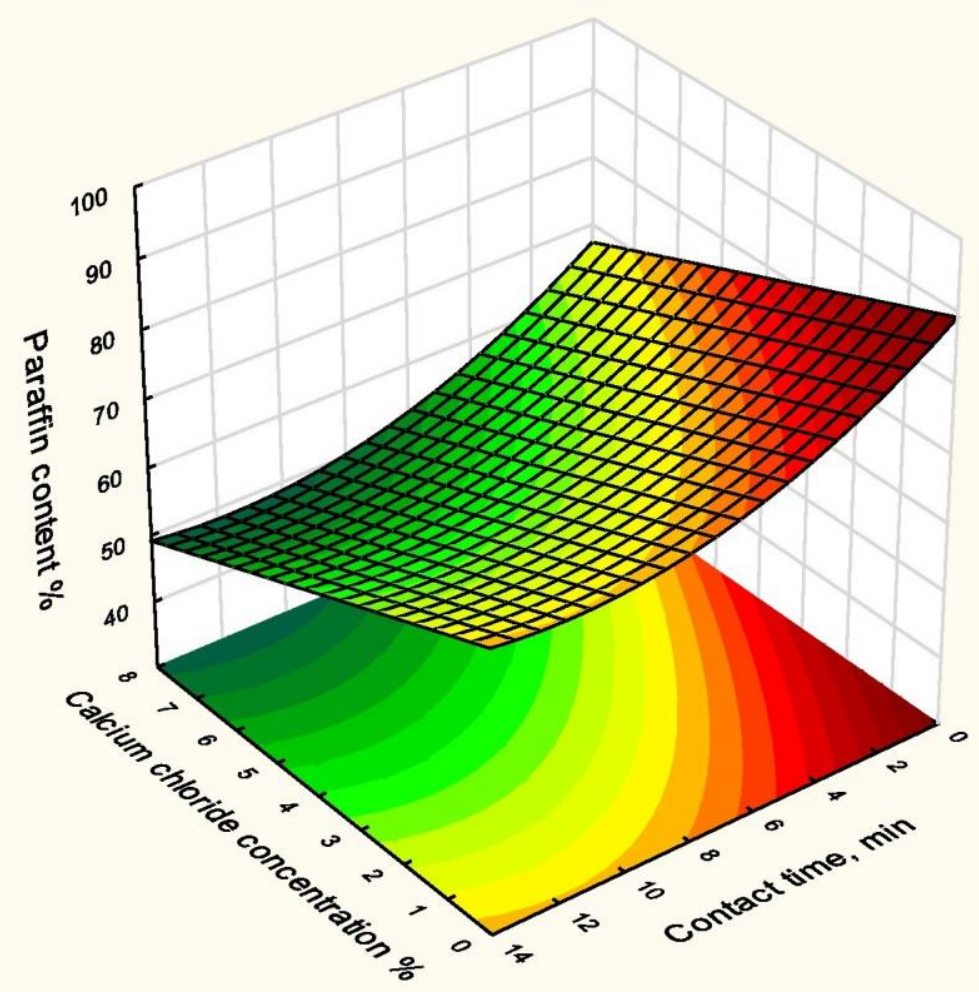

Fig. 4. Variation of paraffin content as a function of $\mathrm{CaCl}_{2}$ concentration and contact time at the medium value $(2.0 \%)$ of sodium alginate concentration.

The effect of the variables on the paraffin content can be expressed by the following equation obtained by multivariate quadratic regression:

$\mathrm{Y}_{\mathrm{par}}=0.6867 * \mathrm{X}_{\mathrm{NaAlg}}-0.2358 * \mathrm{X}_{\mathrm{CaCl} 2}-3.2665 * \mathrm{t}+0.1408 * \mathrm{t}^{2}-1.2517 * \mathrm{X}_{\mathrm{NaAlg}} * \mathrm{X}_{\mathrm{CaCl} 2}+87.8616$

where $\mathrm{Y}_{\mathrm{par}}(\%$,$) is the paraffin content of capsules, \mathrm{X}_{\mathrm{NaAlg}}(\%$,$) is the sodium alginate$ concentration, $\mathrm{X}_{\mathrm{CaCl} 2}(\%$,$) is the calcium chloride concentration, and \mathrm{t}(\mathrm{min})$ is the contact time. The measured paraffin contents were compared with those calculated by the regression equation (Fig. 5), which were in a good agreement with all of the results, the mean quadratic error was $2.58 \%$. 


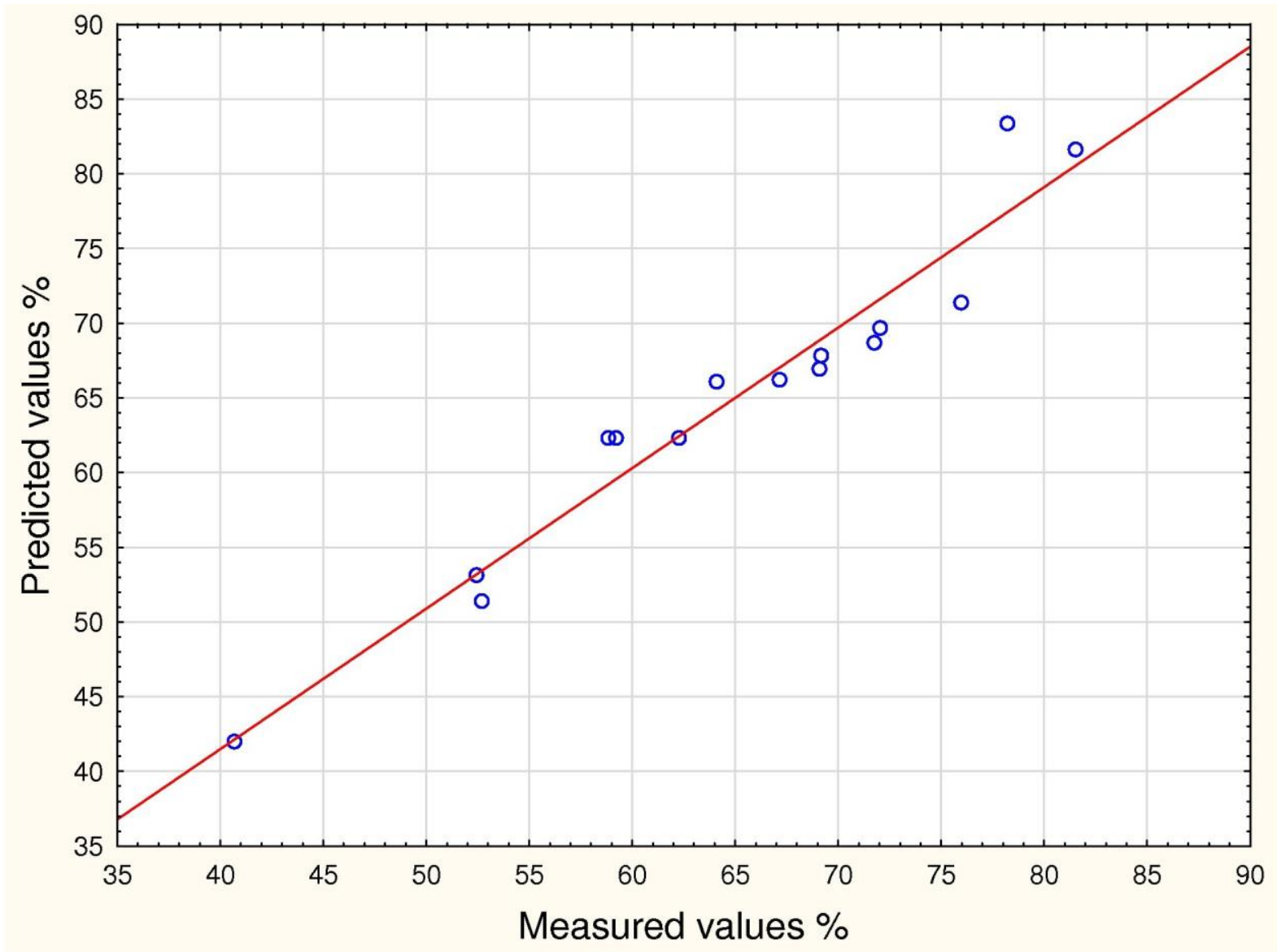

Fig. 5. Comparison of the experimental and predicted paraffin contents.

\subsection{Effect of sodium alginate concentration on paraffin content}

The increasing sodium alginate concentration reduces linearly the paraffin content using any combination of $\mathrm{CaCl}_{2}$ concentration and contact time because of the increasing mass of capsule coating (Fig.6). Maximal paraffin content (85\%) can be achieved by minimal contact time, $\mathrm{CaCl}_{2^{-}}$and sodium alginate concentration. However, under these conditions the capsule wall might not be mechanically stable enough. If the $\mathrm{CaCl}_{2}$ concentration is the lowest, the sodium alginate concentration affects the paraffin content only slightly, since without sufficient $\mathrm{Ca}^{2+}$ necessary for the alginate crosslinking, the coating mass cannot be enlarged. At constant contact time, by increasing the $\mathrm{CaCl}_{2}$ concentration, the slope of the dependency on sodium alginate concentration grew as well. The higher the $\mathrm{Ca}^{2+}$ concentration, the higher is the efficiency to enhance the coating weight and to decrease the ratio of the entrapped paraffin. Using the maximal $\mathrm{CaCl}_{2}$ concentration the highest paraffin content can decrease to as low as $40 \%$, while at the lowest sodium alginate concentration it does not exceed $70 \%$. Applying low or medium value of 
$\mathrm{CaCl}_{2}$ concentration and medium contact time mechanically stable microcapsules with elevated paraffin content $(>70 \%)$ can be formed in the case of low or medium sodium alginate concentration.

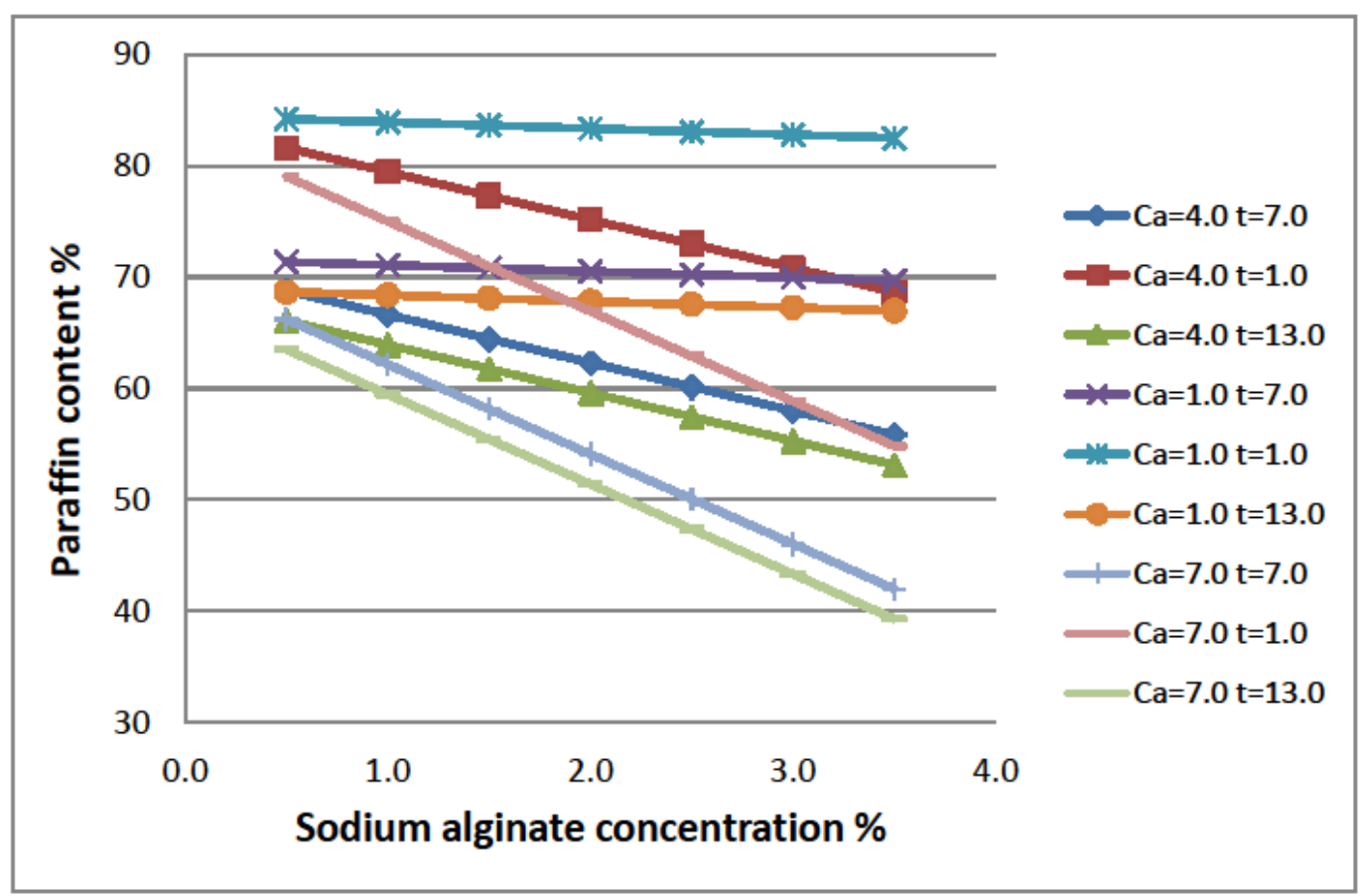

Fig. 6. Effect of sodium alginate concentration on paraffin content at different combinations of $\mathrm{CaCl}_{2}$ concentration and contact time.

\subsection{Effect of $\mathrm{CaCl}_{2}$ concentration on paraffin content}

At fixed sodium alginate concentration and contact time, the capsules paraffin content of the capsules decreases linearly with increasing $\mathrm{CaCl}_{2}$ concentration (Fig.7). This effect is more emphasized with higher sodium alginate concentration and/or higher contact time, when none of them is applied at its lowest value. The highest slope of the change can be carried out by maximal fixed values. The minimal contact time $(1 \mathrm{~min})$ results in the highest paraffin contents independently from the sodium alginate concentration. This latter parameter affected only the slope of the lines, i.e. the highest slope can be reached by the maximal sodium alginate 
concentration. The highest paraffin content achieved by lowest sodium alginate- and $\mathrm{CaCl}_{2}$ concentrations as well as shortest contact time accompanies with mechanically and thermally instable capsules. At fixed medium values of the parameters $\left(\mathrm{X}_{\mathrm{Na}-\mathrm{Alg}}=2.0, \mathrm{t}=7.0 \mathrm{~min}\right)$ or using small sodium alginate concentration and high contact time $\left(\mathrm{X}_{\mathrm{Na}-\mathrm{Alg}}=0.5, \mathrm{t}=13.0 \mathrm{~min}\right)$ in the $\mathrm{CaCl}_{2}$ concentration range of $\mathrm{X}_{\mathrm{CaCl} 2}=2,0-3,0 \%$ the paraffin content was appropriately high (65-68 \% ) with strong capsule coating.

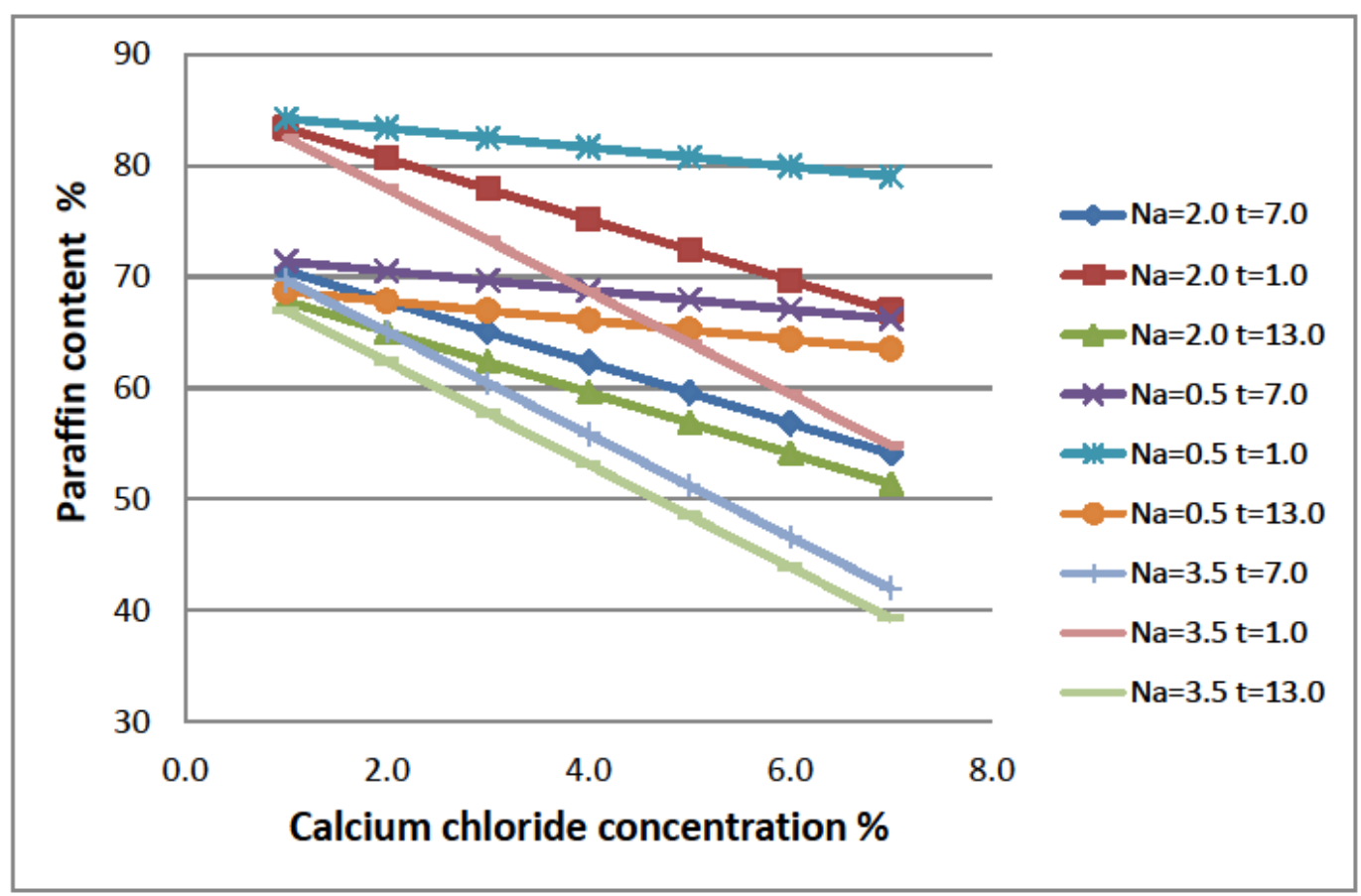

Fig. 7. Effect of $\mathrm{CaCl}_{2}$ concentration on paraffin content at different combination of sodium alginate concentration and contact time.

\subsection{Effect of contact time on paraffin content}

The paraffin content was not linearly dependent on the contact time (Fig. 8). During the first 7 min, the change was steeper, which decreased by longer contact time. The high paraffin content is corresponding to the conditions shown in the previous subsection, in addition using minimal sodium alginate- and medium $\mathrm{CaCl}_{2}$ concentrations is also advantageous. Paraffin content above 
$65 \%$ is available using the following parameters: $\left(\mathrm{X}_{\mathrm{Na}-\mathrm{Alg}}=0.5 \%, \mathrm{X}_{\mathrm{CaCl} 2}=7.0 \%\right.$ and $\mathrm{X}_{\mathrm{NaAlg}}=2.0$ $\%, \mathrm{X}_{\mathrm{CaCl} 2}=4.0 \%$ ), 3-5 min contact time. Above this value there is not any substantial beneficial effect on the paraffin content of the capsules. As represented above though minimal contact time increases the available paraffin content, simultaneously the coating mass might be reduced to a mechanically and thermally instable state. Appropriate sodium alginate concentration (medium level: $2.0 \%$ ) and medium or high $\mathrm{CaCl}_{2}$ concentration (4.0-7.0 \% ) can equilibrate this influence.

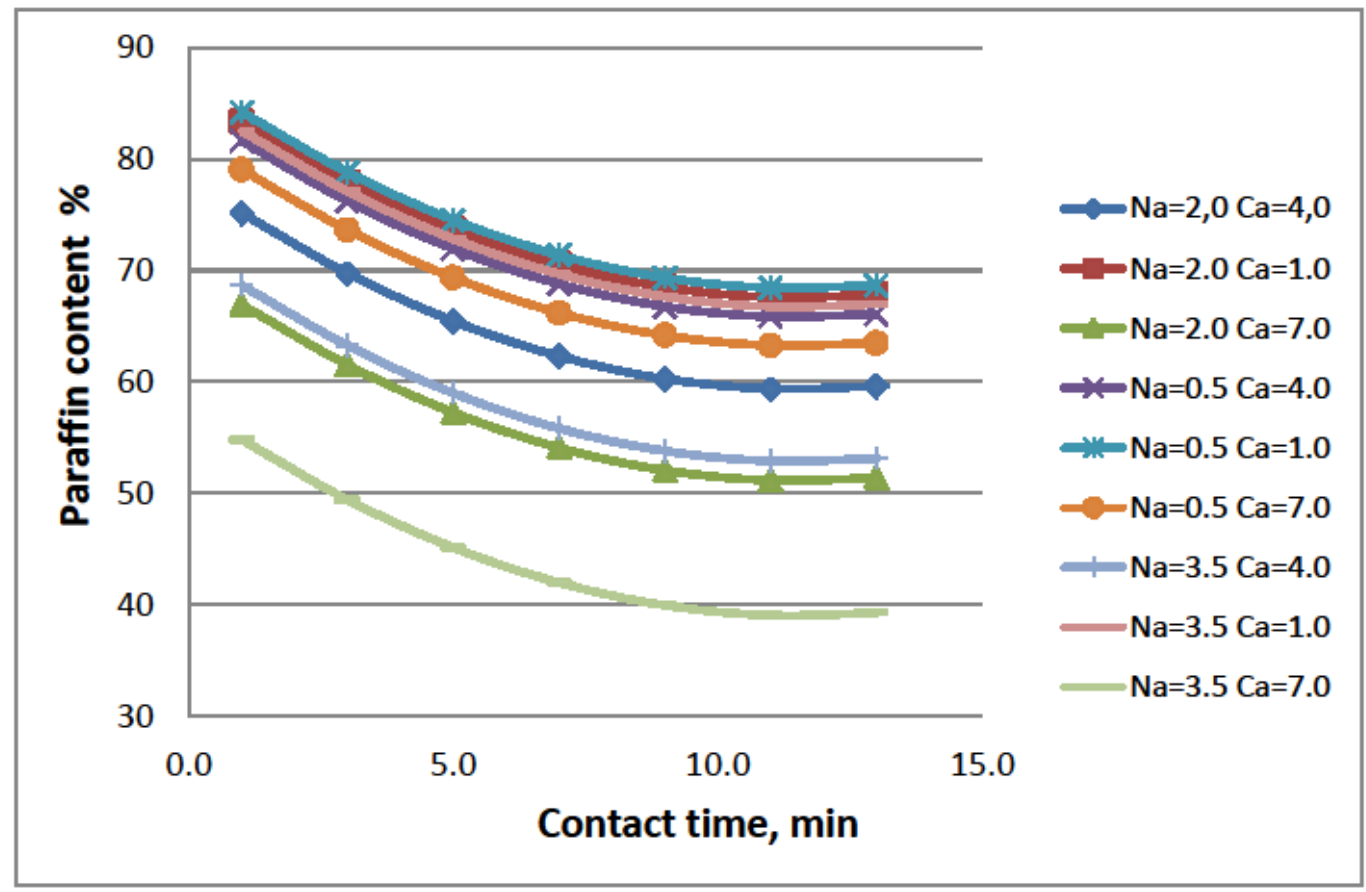

Fig. 8. Effect of contact time on paraffin content at different combination of sodium alginate- and $\mathrm{CaCl}_{2}$ concentration.

\subsection{Effect of sodium alginate quality}

Recently, PCM content of calcium alginate microcapsules was optimized using sodium alginate with low viscosity (Németh et al., 2015). In the present study we found that with encapsulating material of high viscosity the maximum paraffin content can be enhanced enormously from $48 \%$ up to $81.5 \%$. This shows that the quality, especially the viscosity of sodium alginate is of crucial importance. The reason for this phenomenon is that when the viscosity of the entrapping material 
is multiplied, considerably smaller amount of it is sufficient for the emulsification of the paraffin in its solution. The effects of variables were similar in the two studies. Increasing the sodium alginate- and $\mathrm{CaCl}_{2}$ concentration as well as the contact time decreased the paraffin content in both of the studies. Because of the much higher viscosity of the present work significantly lower sodium alginate- and $\mathrm{CaCl}_{2}$ concentrations enabled the emulsification of more paraffin.

\subsection{Coconut oil loaded calcium alginate capsules}

After process optimization using the parameters that promised high PCM loading and appropriate mechanical and thermal stability, coconut oil was incorporated in the microcapsules in five scaled-up parallel experiments. The coconut oil loading measured by PCM extraction and gravimetry was found to be similar to the values optimized using paraffin PCM, i.e. $81.1 \pm 4.6 \%$. SEM images (Fig. 9A) show that spherical microparticles with smooth surface were formed. Compared to the microcomposites prepared recently (Németh et al., 2015) by the low-viscosity sodium alginate in the present work, as a consequence of substantially higher PCM loading, significantly thinner capsule wall was produced (Fig. 9B).

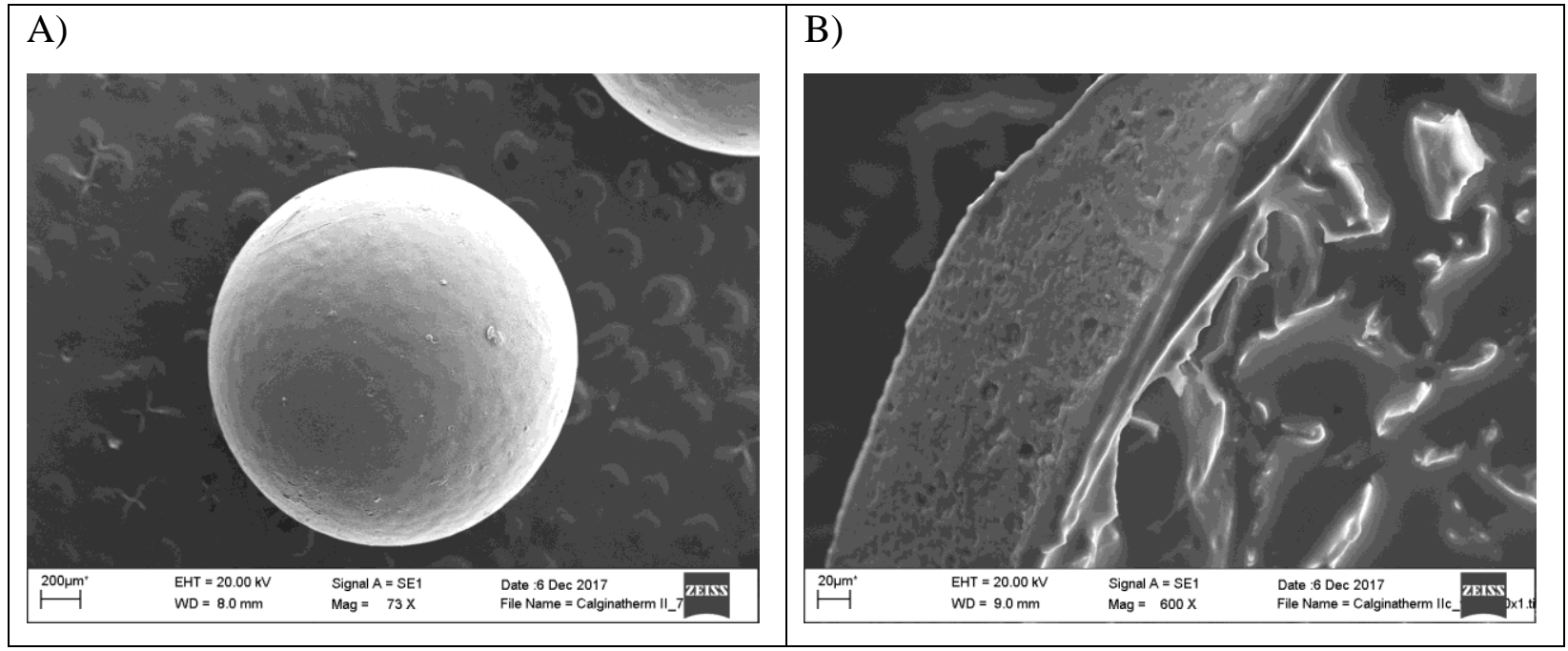

Fig. 9. SEM images of calcium alginate microcapsule containing coconut oil (A) and its crosssection $(\mathrm{B})$.

The thermal behaviour and stability of the capsules was monitored by thermogravimetric analysis. The mass variation (TG) curves of the three raw materials are plotted against temperature (Fig. 10). On the coconut oil's TG curve, two mass loss steps can be seen. The first, largest mass loss occurred between 195 and $426^{\circ} \mathrm{C}$ (mass loss $98.5 \%$ ), which was followed by a 
much smaller step between 426 and $609{ }^{\circ} \mathrm{C}$ (corresponding mass loss 1.3\%). The first step belongs to the oxidative burning of the coconut oil, while the second step, at much higher temperatures, derives from the oxidation of the carbonaceous residue resulted from the incomplete burning of the oil. The total mass loss at the end of the measurement was $99.8 \%$.

For the TG analysis calcium alginate microcapsules without PCM were also prepared. The TG trace of the calcium alginate spheres was much more complicated, than that of the coconut oil. The decomposition of calcium alginate capsules was consisted of 5 steps. From the beginning of the measurement, up to $192.5{ }^{\circ} \mathrm{C}, 6.2 \%$ of physically bound water and some volatiles were released. Above this temperature up to $716{ }^{\circ} \mathrm{C}$, complex oxidative decomposition of the alginate took place. The total mass loss during these three steps was 73.7\%. As a result of the burning of the organic content of the calcium alginate, carbon dioxide and highly reactive calcium oxide formed. At lower temperatures the calcium oxide and carbon dioxide reacted to yield calcium carbonate.

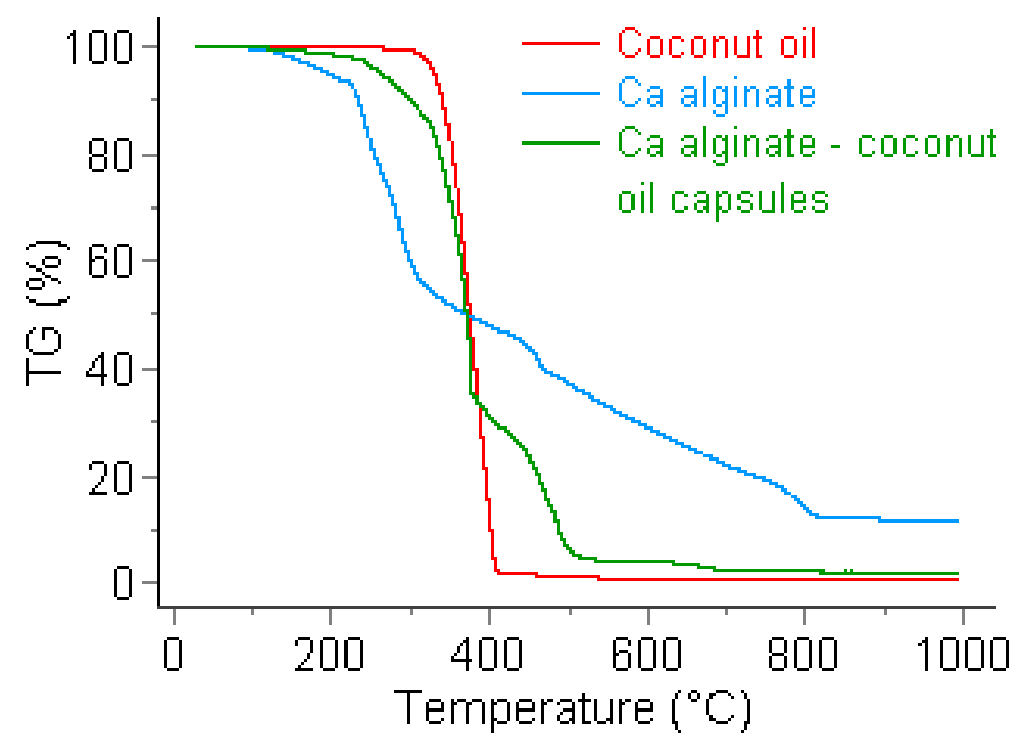

Fig. 10. Thermogravimetric curves of coconut oil, calcium alginate blank capsules and calcium alginate-coconut oil microcapsules.

The as formed calcium carbonate decomposed between 716 and $826^{\circ} \mathrm{C}$, with a mass decrease of $8.3 \%$, resulting again in calcium oxide. The total mass loss during the measurement was $88.1 \%$, the remaining $11.9 \%$ residue corresponds to the amount of calcium oxide. In order to be able to investigate the calcium alginate coated coconut oil composites, a small (500 micrometer) hole 
was drilled in the calcium alginate shell in order to facilitate the release of volatiles from the core. Without this sample preparation step, the microcapsules exploded during the heating, which confirmed that the shell formed a leak-proof layer around the core. The TG curve of the composite capsules laid between the two curves of the two components, since it involved the properties of both of the coconut oil and the calcium alginate. From 30 to $207^{\circ} \mathrm{C}$, a much lower mass loss $(1.9 \%)$ was observed compared to the pure calcium alginate, which suggests that the microcapsules contained much lower amount of water and other volatiles. In the second step (207 and $298{ }^{\circ} \mathrm{C}$, mass loss $9.9 \%$ ), a gentle degradation of the calcium alginate shell started, while above $298{ }^{\circ} \mathrm{C}$ up to $409{ }^{\circ} \mathrm{C}$ a much higher and faster mass loss can be seen $(59.5 \%)$, which corresponds to the volatilization and burning of the coconut oil core. The complete burning of the alginate shell took place between 410 and $544.5{ }^{\circ} \mathrm{C}$, resulting in a mass loss of $24.4 \%$. Above $544.5^{\circ} \mathrm{C}$ another small mass loss step can be observed, which arises from the decomposition of the formed calcium carbonate, as it was described previously. It can be noted that in the case of composite microspheres the calcium carbonate decomposed at much lower temperatures compared to that found in pure calcium alginate. The reason is that the organic content of the composite microspheres burnt with the release of considerably higher amount of heat and gases (mainly carbon dioxide), thus the as formed reactive, nanoparticles of calcium carbonate decomposed at considerably lower temperatures. It can also be noted, that the amount of inorganic residue (calcium oxide) left at the end of measurement was much lower in the case of microspheres $(2.2 \%)$, compared to the pure $\mathrm{Ca}$ alginate $(11.9 \%)$, which indicated the calcium content of the microspheres was lower, since they contained a high ratio of PCM beside the calcium alginate.

The heat storing capacity of the coconut oil and the calcium alginate-PCM microparticles was analysed by differential scanning calorimetry (Fig. 11). The melting and freezing enthalpy changes were found to be $115.3 \mathrm{~J} / \mathrm{g}$ and $108.1 \mathrm{~J} / \mathrm{g}$, respectively, for the pure coconut oil (Fig. 11a). The corresponding heat storage capacities of calcium alginate microcapsules containing coconut oil were $84.7 \mathrm{~J} / \mathrm{g}$ and $84.5 \mathrm{~J} / \mathrm{g}$, respectively (Fig. 11b), which were in a good agreement with PCM content determined by chemical extraction and gravimetry. 


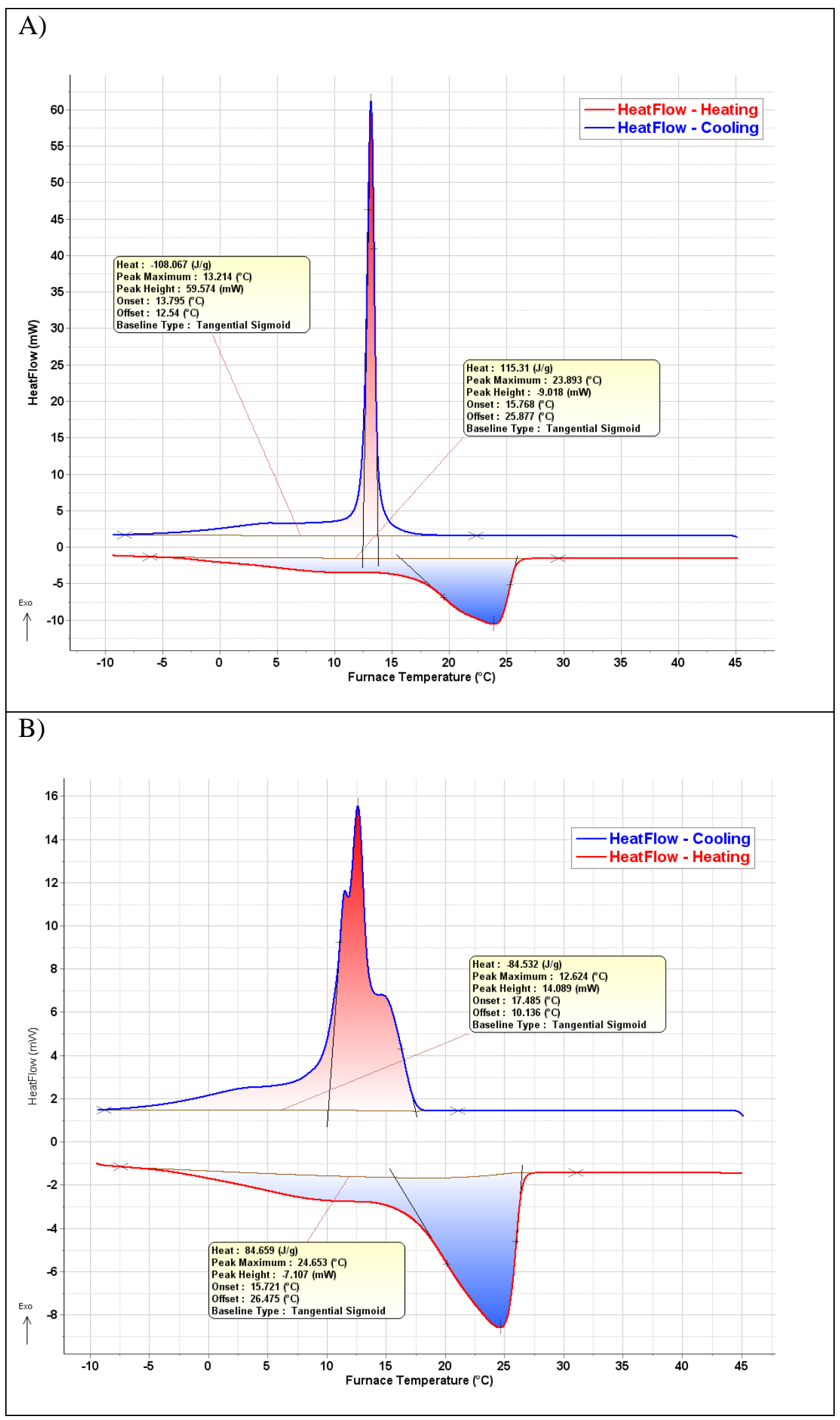


Fig. 11. DSC measurement results of pure coconut oil (A) and that of coconut oil containing microcapsules (B).

\section{Conclusions}

A recently developed process for the preparation of calcium alginate microcapsules containing paraffin PCM was significantly improved by applying a highly viscous sodium alginate carrier. The optimization of the procedure was supported by experimental design and statistical evaluation. As a maximum $81.5 \%$ paraffin content was realized in the calcium alginate capsules that were characterized by monodisperse size distribution. Furthermore, by using coconut oil as PCM instead of paraffin in the composites, we managed to prepare completely bio-originated latent heat storing spherical microcapsules with accordingly high average PCM loading (81.1\%) in a scaled-up procedure. The high coconut oil content was reflected also in the melting $(84.7 \mathrm{~J} / \mathrm{g})$ and freezing $(84.5 \mathrm{~J} / \mathrm{g})$ latent heat capacities in comparison with these values for pure oil, which were $115.3 \mathrm{~J} / \mathrm{g}$ and $108.1 \mathrm{~J} / \mathrm{g}$, respectively. Beside the advantages of the bio-based PCMs mentioned in the introduction, the main disadvantage, that is, their lower heat storing capability must be mentioned. In further studies the microencapsulation of an optimal bio-originated PCM is aimed, which possesses significantly higher heat capacity, consequently the heat storage of the environmentally friendly PCM capsules could be further improved. Considering the fact that the preparation process originally optimized for paraffin enabled the production of microcapsules incorporating coconut oil that has significantly different chemical character, probably this latent heat storing microparticulate system can be applied for microencapsulation of other bio-PCMs which have significantly higher heat capacity, e.g. saturated fatty acids that are the main components of coconut oil.

\section{Acknowledgement}

The research within project No. GINOP-2.2.1-15-2016-00010 and VEKOP-2.3.2-16-2017-00013 was supported by the European Union and the State of Hungary, co-financed by the European Regional Development Fund.

\section{References}


Cao, L., Tang, F., Fang, G., 2014. Preparation and characteristics of microencapsulated palmitic acid with $\mathrm{TiO} 2$ shell as shape-stabilized thermal energy storage materials. Sol. Energy Mater. Sol. Cells. 123, 183-188.

Doguscu, D.K., Altintas, A., Sari, A., Alkan, C., 2017. Polystyrene microcapsules with palmiticcapric acid eutectic mixture as building thermal energy storage materials. Energ. Buildings. 150 (2017) 376-382.

Fang, X., Hao, P., Song, B., Tuan, C.C., Wong, C.P., Yu. Z.T., 2017. Form-stable phase change material embedded with chitosan-derived carbon aerogel. Mater. Letters. 195, 79-81.

Feczko, T., Trif, L., Horak, D., 2016 Latent heat storage by silica-coated polymer beads containing organic phase change materials. Solar Energy, 132, 405-414.

Hu, X., Huang, Z., Yu, X., Li, B., 2013. Preparation and thermal energy storage of carboxymethyl cellulose-modified nanocapsules. Bioenerg. Res. 6, 1135-1141.

Jeong, S.G., Chung, O., Yu, S., 2013. Improvement of the thermal properties of Bio-based PCM using exfoliated graphite nanoplatelets. Sol. Energy Mater. Sol. Cells. 117, 87-92.

Kang, Y., Jeong, S.G., Wi, S., 2015. Energy efficient Bio-based PCM with silica fume composites to apply in concrete for energy saving in buildings. Sol. Energy Mater. Sol. Cells. 143, 430-434.

Konuklu, Y., Paksoy, H.O., Unal, M., Konuklu, S., 2014. Microencapsulation of a fatty acid with Poly(melamine-urea-formaldehyde. Energ. Convers. Manage. 80, 382-390.

Konuklu, Y., Unal, M., Paksoy, H.O., 2014. Microencapsulation of caprylic acid with different wall materials as phase change material for thermal energy storage. Sol. Energy Mater. Sol. Cells. 120, 536-542.

Konuklu, Y., Paksoy, H.O., 2017. Polystyrene-based caprylic acid microencapsulation for thermal energy storage. Sol. Energy Mater. Sol. Cells. 159, 235-242.

Li, W., Zhang, X.X., Wang, X.C., Tang, G.Y., Shi, H.F., 2012. Fabrication and morphological characterization of microencapsulated phase change materials (MicroPCMs) and macrocapsules containing MicroPCMs for thermal energy storage. Energy. 38, 249-254.

Li, W., Zhang, R., Jiang, N., Tang, X.F., Shi, H.F., Zhang, X.X, Zhang, Y., Dong, L., Zhang, N., 2013. Composite macrocapsule of phase change materials/expanded graphite for thermal energy storage. Energy. 57, 607-614. 
Liang, W.G., Yang, C., Wen, G.Q., Wang, W., Ju, X.J., Xie, R., Chu, L.Y. A facile and controllable method to encapsulate phase change materials with non-toxic and biocompatible chemicals. Appl. Therm. Eng. 70 (2014) 817-826.

Luo, J., Zhao, L., Yang, Y., Song, G., Liu, Y., Chen, G. Tang, 2016. Emulsifying ability and cross-linking of silk fibroin microcapsules containing phase change materials. Sol. Energy Mater. Sol. Cells, 147, 144-149.

Nemeth, B., Nemeth, A.S., Toth, J., Fodor-Kardos, A., Gyenis, J., Feczko T., 2015. Consolidated microcapsules with double alginate shell containing paraffin for latent heat storage. Sol. Energy Mater. Sol. Cells. 143, 397-405.

Salunkhe, P.B., Shembekar, P.S., 2012. A review on effect of phase change material encapsulation on the thermal performance of a system. Renew. Sust. Energ. Rev. 16, $5603-5616$.

Sarı, A., Karaipekli, A., 2007. Thermal conductivity and latent heat thermal energy storage characteristics of paraffin/expanded graphite composite as phase change material. Appl. Therm. Eng. 27, 1271-1277.

Serale, G., Goia F., Perino, M., 2016. Numerical model and simulation of a solar thermal collector with slurry Phase Change Material (PCM) as the heat transfer fluid. Solar Energy, 134, 2016, 429-444.

Wang, J.P., Zhang, X.X., Wang, X.C., 2011. Preparation, characterization and permeation kinetics description of calcium alginate macro-capsules containing shape-stabilize phase change materials. Renew. Energy. 36, 2984-2991.

Zhang, T., Wang, Y., Shi, H., Yang, W., 2012. Fabrication and performances of new kind microencapsulated phase change material based on stearic acid core and polycarbonate shell. Energ. Convers. Manage. 64, 1-7. 